\title{
Los 144.000 y su relación con la gran multitud a la luz de los escritos de Elena G. de White
}

MAICOL CORTES ${ }^{1}$

Resumen: En este estudio se analizarán elementos de los capítulos 7 y 14 del libro de Apocalipsis, lugar en donde Juan describe a los 144.000 y la gran multitud. El previo análisis se hará con la finalidad de presentar la relación que existe entre los 144.000 y la gran multitud, para responder a la interrogante sobre la simbología o literalidad del primer grupo. Además, se examinarán algunos textos de Elena de White para ver si la descripción de los detalles que ella narra coincide con las conclusiones presentadas en este análisis. Aunque este estudio considera ciertas características de la gran multitud, se centrará en los 144.000 .

Palabras clave: Apocalipsis; 144.000; gran multitud; Elena G. de White.

\section{The 144.000 and their relationship with the great multitude in light of the writings of Ellen $G$. White}

\begin{abstract}
This article analyses the narrative of the great multitude in Revelation 7 and 14. This analysis aims to describe the relationship between the 144,000 and the great multitude. In answering the question about the symbolism or the literality of the 144,000 , this group will be compared with some of Ellen White's texts. Although this study considers some characteristics of the great multitude, it will focus on the 144,000.
\end{abstract}

Keywords: Revelation; 144.000; Great Multitude; Ellen G. White.

La identidad de los 144.000 y su relación con la gran multitud es uno de los tantos temas de interés para los lectores del libro de Apocalipsis. A pesar de la variedad de interpretaciones

\footnotetext{
1 Maicol Cortés se encuentra actualmente estudiando una maestría en Estudios Judíos en The Hebrew University of Jerusalem (Rotherberg International School). Cortés es Magister en Teología (Nuevo Testamento) en The Adventist International Institute of Advanced Studies (AIIAS) en Silang Cavite, Filipinas y posee una licenciatura en teología por la Universidad Adventista de Chile. E-mail: maicol.cortes@mail.huji.ac.il
} 
relacionadas con estos grupos, ${ }^{2}$ hay unanimidad entre los estudiosos quienes consideran que los 144.000 son un número simbólico. El argumento para esta conclusión, entre otros, es la pertenencia de este grupo a "las tribus de los hijos de Israel" (Ap 7:4), expresión usada por los autores del Nuevo Testamento (NT) para referirse a la iglesia cristiana y no al Israel literal (MOUNCE, 1998, p. 158; Ro 2:29; Ga 6:16; Sant 1:1).

El objetivo de este estudio es analizar algunos elementos importantes en la narrativa de Apocalipsis 7 y 14, lugar en donde se describen los 144.000 y la gran multitud. Para ello, se considerarán las características de este grupo presentadas en Apocalipsis (Ap 14:1-5). El previo análisis se hace con la finalidad de presentar la relación que existe entre ambos grupos, para responder a la interrogante sobre la simbología o literalidad de los 144.000. Además, se examinarán algunos textos de Elena G. de White para ver si la descripción de los detalles que ella narra coincide con las conclusiones presentadas en este análisis.

\section{El contexto en el que se hallan los 144.000}

La presentación de los 144.000 en el capítulo 7 parece responder a la pregunta formulada en Apocalipsis 6:17 “porque el gran día de su ira ha llegado y ¿quién podrá sostenerse en pie?” Dado que el capítulo 6 presenta la segunda venida de Cristo, parece lógico pensar que los 144.000 son un grupo que se constituye cerca del final de la historia de este mundo. Algunos elementos en la narrativa de Apocalipsis 7 parecen apoyar esta conclusión, tal como se verá a continuación.

Apocalipsis 7:1-3 nos dice que un ángel poderoso ordena a otros cuatro ángeles, que estos no deben dañar "la tierra, el mar y los árboles" ya que los "siervos de Dios" aún no han sido sellados. A este grupo especial de sellados se les conoce como los 144.000 (Ap 7:4).

La frase tēn gèn, mète tèn thalassan, mète ta dendra "la tierra, el mar y los árboles" puede tener una doble función. En primer lugar, esta expresión podría señalar entidades humanas corrompidas por el pecado. Los árboles son a menudo identificados en la Biblia con seres humanos (S1 1:1-3; Jr 17:7-8; Dn 4:20-22). En Apocalipsis 12:9, el dragón fue arrojado a la tierra (gēn) luego de perder la batalla en el cielo contra Miguel, pero además se nos dice “ ¡Ay de los moradores de la tierra y del mar! [tēn gèn kai tèn thalassan], porque el diablo ha descendido a vosotros con gran ira, sabiendo que tiene poco tiempo" (Ap 12:12). Parece ser que después de corromper el cielo, el dragón hace lo mismo con la tierra y el mar. Por ello, no es de extrañar que desde estos dos elementos surjan poderes antagónicos al gobierno de Dios (Ap 13:1, 11).

En segundo lugar, la frase "la tierra, el mar y los árboles" podría ser una referencia a la naturaleza que será afectada por las plagas. Por ejemplo: el primero de los siete ángeles "derramó su copa sobre la tierra [tēn gēn], y vino una úlcera maligna" (Ap 16:2). El segundo lo hace "sobre el mar [tèn thalassan], y éste se convirtió en sangre como de muerto" (Ap 16:3). Finalmente, la cuarta plaga afecta al sol el cual quema a los hombres (Ap 16:8). Las plagas afectan a la naturaleza, así como a la humanidad en su conjunto (STEFANOVIĆ, 2013, p. 477-478).

Si se considera la evidencia anterior, se podría establecer que los 144.000 son un grupo especial de personas que estarán vivas ante el inminente regreso de Cristo y son protegidas por él de los desastres previos a su venida.

2 Véase la sección "La relación entre ambos grupos". 


\section{La relación entre ambos grupos}

Variadas son las interpretaciones que buscan explicar la identidad de los 144.000 y la relación de este grupo con la gran multitud, hay por lo menos tres explicaciones presentadas por los eruditos. ${ }^{3}$ El primer punto de vista considera que los 144.000 y la gran multitud serían un mismo grupo, pero presentado en diferentes escenarios. Los 144.000 son los fieles que recibirán el sello de Dios, pero luego son presentados como una gran multitud adorando en el trono de Dios. La segunda posición considera que los 144.000 y la gran multitud son grupos diferentes. El primero es un grupo especial de redimidos (las primicias), mientras que el segundo está constituido por los redimidos de todas las épocas. Finalmente, la tercera perspectiva cree que la gran multitud representa a la totalidad de los redimidos, lo que incluye a los 144.000 (NICHOL et al., 1995, p. 799-800). La pregunta es ¿cuál de estas tres posiciones es la más cercana al texto bíblico? Para responder a esta pregunta a continuación se analizarán algunos elementos internos y externos de la narrativa de Apocalipsis.

\section{El uso de la frase "oí" y "vi"}

Juan usa una técnica literaria en donde aquello que él "oye" no es lo mismo que luego "ve". Por ejemplo, Juan oye a su espalda una voz como de trompeta (Ap 1:10), pero cuando él da la vuelta, lo que ve no es una trompeta sino "siete candelabros de oro" y a Jesús en medio de ellos (Ap 1:12-13). Luego en el capítulo 5 Juan oye acerca del "León de la tribu de Judá, la raíz de David”, pero el profeta ve a "un Cordero como inmolado" (Ap 5:6). En Apocalipsis 17:3, Juan oye acerca de una mujer "que está sentada sobre muchas aguas", sin embargo, él ve "a una mujer sentada sobre una bestia escarlata" (STEFANOVIĆ, 2013, p. 29). Al parecer esta técnica literaria es repetida por Juan en Apocalipsis 7, ya que él primeramente "oye el número de los sellados" (Ap 7:4), pero ve a una "gran multitud" (Ap 7:9). Si esta conclusión es correcta, eso quiere decir que los 144.000 deben ser interpretados como un número simbólico entendiendo que ambos grupos (los 144.000 y la gran multitud) son uno. Es interesante notar que la frase èkousa ton arithmon "oí el número" (Ap 7:4) se repite en Apocalipsis 9:16, lugar en donde Juan oye el número de los opositores al gobierno de Dios (doscientos millones). Esta última cifra también es simbólica y sirve para enfatizar la diferencia numérica entre ambos grupos (Cf. Ap 20:8).

La técnica literaria "oí y vi" presentada en los capítulos 5 y 7 de Apocalipsis, parece enfatizar la conexión existente entre los 144.000 y la gran multitud. Los primeros dos títulos aplicados a Jesús en Apocalipsis 5:5 son "el León de la tribu de Judá y la raíz de David", los cuales se enfocan en un Mesías militar que conquista a los enemigos de su pueblo (Gn 49:9; Is 11:1-5) (BEALE, 2015, p. 114). Sin embargo, Juan ve a un Cordero, símbolo que señala la victoria por medio de la muerte sacrificial de Cristo (Ex 12:5-7; Is 53:7). Entonces parece ser que Juan describe que la victoria final será alcanzada no por medio de fuerza militar - una idea presente en la mentalidad judía (Mt 26:51-54; 27:38-41; Lc 23:35-38; In 19:15, 17-21), sino más bien por medio del sacrificio de Jesús.

El León y el Cordero señalan a un mismo personaje (Jesús), así como los 144.000 y la gran multitud apuntan a un mismo grupo de personas. ${ }^{4}$ Lo interesante es que el León presenta algunas

\footnotetext{
3 Para ver una evaluación completa de las diferentes propuestas véase (MENDOZA, 2011, p. 49-51)

4 Véase la discusión bajo el título "El uso de la frase oí y vi".
} 
similitudes con los 144.000, mientras que, el Cordero lo hace con la gran multitud. Por ejemplo, ho leōn ho ōn ek tēs phylēs Iouda "el León de la tribu de Judá" (Ap 5:5) encuentra su símil en la tribu de Judá mencionada en el capítulo 7 (ek phylēs Iouda). Por otra parte, el Cordero ha redimido a "todo linaje, lengua, pueblo y nación" haciéndoles un reino de sacerdotes (Ap 5:9-10), así como la gran multitud es un grupo perteneciente a "todas las naciones, tribus, pueblos y lenguas" los cuales adoran ante el Cordero y sirven día y noche en el templo de Dios (Ap 7:9, 15) (BAUCKHAM, 1998, p. 216).

Estas similitudes indicarían que los 144.000 son la armada espiritual del león Jesús, mientras que la gran multitud revelaría que la victoria se logró no por la fuerza, sino a través del sacrificio del Cordero. Esta conclusión estaría en armonía con las palabras de Jesús (Mt 26:51-54) y con la descripción de la última batalla, lugar en donde se enfrentarán las armadas del Cordero y el Dragón (Ap 16:12-14). Además, el censo de las tribus de Israel, tal como se hace con los 144.000, estaba generalmente relacionado al poderío militar ( Nm 1:26; 2 Sam 24) y se censaban solo a los varones en edad militar (Nm 1:3, 18, 20; 1 Cr 27:23 Cf. Ap 14:4). Por lo tanto, no es de extrañar que Juan mencione que este es un grupo de vírgenes ya que no se han contaminado con mujeres - un requisito que debían cumplir todos los hombres de guerra en Israel (1 Sam 21:5; 2 Sam 11:11; Cf. Ap 14:4; 17:5).

\section{Los 144.000 y sus características}

Las características de los 144.000 también favorecen una lectura simbólica de este número, considerando que este grupo tiene una segunda mención en el capítulo 14 - lugar en donde se señalan explícitamente algunas de sus cualidades. Se examinará brevemente las características de este grupo presentadas en este último capítulo, para luego comentar aquellas mencionadas en el capítulo 7, dado a que el espacio no permite un estudio detallado, el siguiente análisis comprenderá solo algunas cualidades mencionadas en ambos capítulos.

\section{Comprados de entre los de la tierra}

La frase ègorasmenoi apo tēs gēe "fueron comprados de entre los de la tierra" (Ap 14:3 SRV-BRG), ${ }^{5}$ enfatiza al menos dos elementos. En primer lugar, el verbo agorazō (comprar) (MOUNCE, 2006, p. 91) aparece en seis ocasiones en Apocalipsis, en tres de ellas esta palabra se relaciona con la redención que el Cordero efectúa a través de su sangre en la vida del creyente (Ap 5:9, 14:3-4). En segundo lugar, se menciona que este grupo será redimido de entre aquellos que moran en la tierra. La frase "los moradores de la tierra" está siempre relacionada con los incrédulos (Ap $6: 10 ; 8: 13 ; 11: 10 ; 13: 8,12,14 ; 14: 6 ; 17: 2,8)$. Por lo tanto, la expresión "fueron comprados de entre los de la tierra" se refiere a que los 144.000 son un grupo redimido de entre los incrédulos de la tierra, los cuales son comprados/rescatados por la sangre de Jesús (1 Cor 6:20 Cf. Ap 5:9).

\footnotetext{
5 SRV-BRG, Spanish Blue Red and Gold Letter edition.
} 


\section{No se contaminan con mujeres pues son vírgenes}

La expresión "éstos son los que no se han contaminado con mujeres pues son vírgenes" (Ap 14:4) tiene al menos dos importantes connotaciones: (1) nos revela el carácter simbólico de este grupo y (2) nos dice que los 144.000 serán protagonistas de las escenas finales de este mundo. Si los 144.000 fueran literales, entonces eso quiere decir que debe ser un grupo compuesto solo por varones. No obstante, el lenguaje simbólico de Apocalipsis nos presenta otra realidad.

El verbo molunō "contaminarse" puede señalar una contaminación de la conciencia (1 Cor 8:7) o de las vestiduras (Ap 3:4), esto último está relacionado al carácter del cristiano "El vencedor será vestido de vestiduras blancas" (Ap 3:5) (STEFANOVIĆ, 2013, p. 140-143). Además, el verbo "contaminarse" es un aoristo; esto quiere decir que la descripción de la no contaminación de los 144.000 por medio de mujeres describe un evento específico. Probablemente aquel acontecimiento se refiere a las últimas pruebas por las cuales tendrán que pasar los cristianos, momento en donde tendrán que ser fieles a Dios rechazando las enseñanzas de la mujer ramera y sus hijas (Ap 17:1-2,6).

El sustantivo gyne "mujer" aparece 19 veces en Apocalipsis, en ocasiones se refiere a una mujer fiel (Ap 12:1, 4, 6, 13-17; 19:7; 21:9) y en otras a una infiel (Ap 2:20; 17:3-4, 6-7, 9, 18). Los 144.000 no se contaminan con gynaikōn "mujeres", expresión que probablemente se refiere a las rameras mencionadas en el capítulo 17 "Babilonia la grande, la madre de las rameras". El sustantivo pornōn "rameras" pertenece a una familia de cinco palabras (tres sustantivos y dos verbos) (MUELLER, 2001, p. 1) y su significado simbólico se haya restringido al libro de Apocalipsis (MUELLER, 2001, p. 3). Por ejemplo, en la iglesia de Tiatira se menciona que Jezabel seduce a los hijos de Dios a porneuo "fornicar". La fornicación aparece a menudo en el Antiguo Testamento (AT) relacionada con la infidelidad/adulterio a Dios, producto de la idolatría (Is 57:3; Jr 3:8-9). Los 144.000 contrastan con los moradores y reyes de la tierra, los cuales han fornicado con la ramera (Ap 17:1-5). Por ello, Juan describe a este grupo como un conjunto de personas que se mantienen vírgenes; es decir, cristianos que se conservan fieles al cordero Jesús. ${ }^{6}$

En conclusión, la frase "éstos son los que no se han contaminado con mujeres pues son vírgenes" se refiere a que este grupo no contamina su carácter/mente con las falsas doctrinas de la ramera. Por ello, los 144.000 se mantienen fieles a Dios (vírgenes) sin ceder a la idolatría a la que se verán forzados por los enemigos de Dios (Ap 13:11-17).

\section{Han salido de la gran tribulación}

Ya hemos señalado que los 144.000 y la gran multitud son el mismo grupo, pero presentado en diferentes escenarios. La frase Ohutoi eisin hoi erchomenoi ek tēs thlipseōs tēs megalēs "estos son los que han salido de la gran tribulación" (Ap 7:14) es una referencia a la gran multitud en su fase militante, lo cual alude a los 144.000 y su lucha contra la idolatría (ramera y bestias) y los juicios de Dios (las plagas). ${ }^{7}$ La expresión "no tendrán hambre ni sed, y el sol no caerá más sobre

\footnotetext{
${ }^{6}$ Craig R. Koester también ve la posibilidad de entender el término "vírgenes" como una alusión de que este grupo de cristianos (los 144.000$)$ serán parte de la "esposa del Cordero" o "la nueva Jerusalén”. Aquella iglesia sin mácula que Cristo viene a desposar (Ap 12:1; 19:7-8; 21:2). Ellos deben mantenerse puros, así como las doncellas debían hacerlo para su esposo (Dt 22:13-21) (KOESTER, 2014, p. 611). Esta conclusión tendría sentido si se considera que Apocalipsis enfatiza las bodas del Cordero como un evento trascendental (Ap 19:7, 9), el que es descrito a modo de parábola por el mismo Jesús en los evangelios (Mt 22:2-4; 25:1-13; Lc 12:35-36).

7 Para ver la relación de los 144.000 y las plagas, véase la cita de Elena de White bajo el título "Los 144.000 y la gran multitud".
} 
ellos, ni calor alguno" (Ap 7:16) apoya la conclusión previa, puesto que en el cielo (lugar en donde se encuentra la gran multitud) no se sufrirá nada de aquello. Por otra parte, el texto nos dice que este grupo pasará por la gran tribulación (tês thlipseōs tēs megalēs). Aquella prueba predicha por el profeta Daniel "En aquel tiempo se levantará Miguel, el gran príncipe que está de parte de los hijos de tu pueblo. Será tiempo de angustia [hèmera thlipseōs], cual nunca fue desde que hubo gente hasta entonces" (Dn 12:1 LXX Cf. Ap 3:10). Daniel concluye que en aquel tiempo el pueblo de Dios sería librado, dicha liberación se logra en Apocalipsis por medio de Jesús (Ap 16:12-16; $19: 1-2,6-8,19-21 ; 20: 7-10)$.

\section{Estos fueron redimidos de entre los hombres como primicias}

La frase houtoi ègorasthēsan apo tōn anthrōpōn "estos fueron redimidos/comprados de entre los hombres" (Ap 14:4), tiene gran similitud con la expresión hoi ègorasmenoi apo tēs gēe "fueron comprados de entre los de la tierra" (Ap 14:3). Por ello, podríamos suponer que el sustantivo anthrōpōn es equivalente a gēs, dado que en ambas oraciones les antecede la preposición apo. El término anthrōpos aparece 23 veces en Apocalipsis, de las cuales 16 se refieren a los incrédulos. Es decir, Apocalipsis 14:3 nos dice que los 144.000 fueron redimidos/comprados de entre los de la tierra, mientras que, el verso 4 nos dice que fueron redimidos de entre los hombres. Por lo tanto, en ambos casos los 144.000 son redimidos/comprados de entre los impíos, señalados en Apocalipsis como "hombres" o "habitantes de la tierra".

Finalmente, a este grupo de redimidos se les conoce como las primicias. La palabra aparchē "primicias" en la Septuaginta (LXX) está relacionada con la cosecha que debían presentar los penitentes en el santuario (Éx 23:19; Lev 23:10 etc.). Por otra parte, en Santiago leemos que los redimidos son considerados "primicias" (Sant 1:18). La relación entre las primicias/cristianos y el lenguaje de la cosecha no debería de sorprendernos, puesto que, Jesús en los evangelios en reiteradas ocasiones habló de los humanos y sus decisiones en términos agrícolas (Mt 9:37-38; 13:1-9; 20: 1-16; 21:33-44). El lenguaje agrario, también se ve reflejado en Apocalipsis en donde los " 144.000 son redimidos o comprados como las primicias de la cosecha del tiempo del fin (la cosecha del trigo, Ap 14:14-16), en contraste con 'las naciones incrédulas a punto de ser juzgadas' (la cosecha de uvas, Apoc.14:17-20)" (STEFANOVIC, 2013, p. 445).

\section{Son sin mancha}

La última de las características de los 144.000 nos dice que estos amōmoi eisin "son sin mancha”. El adjetivo amōmoi aparece siete veces en el NT, en cinco ocasiones se relaciona con la condición que Dios espera que alcancen los cristianos/iglesia (Ef 1:14; 5:27; Col 1:22; Jds 24; Ap 14:5). Una condición sin amōmoi "mancha", la que se logrará por medio de la muerte de Cristo (Col 1:22). Las otras dos ocasiones en donde aparece la palabra amōmoi se relaciona con Jesús (Heb 9:14; 1 Pe 1:19), lo cual estaría en armonía con el estado que alcanzarán los 144.000; puesto que ellos son los que siguen al Cordero y han lavado sus ropas en Su sangre (Ap 7:14 Cf. 14:4).

8 Véase la discusión sobre la frase "los moradores de la tierra" bajo el título "Comprados de entre los de la tierra". 
La palabra amōmoi aparece en la LXX relacionada con el adjetivo hebreo tāmî̀m, que es el término usado en las actividades del santuario y que describía la condición sin mancha que debían presentar los animales en el sacrificio (MOUNCE, 2006, p. 234) (Lv 3:1, 6; 4:23, 28; Nm $6: 14 ; 28: 3$ ), animales que representaban al cordero Jesús que quitaría el pecado del mundo (Juan 1:29). Por otra parte, el término amōmoi aparece en la LXX relacionado con Abraham y Job. La Escritura describe que a Abraham, Dios le mandó ser "perfecto", mientras que Job "era un hombre perfecto y recto, temeroso de Dios y apartado del mal" (Gn 7:1; Job 1:1); características, que como vimos, Dios espera también de su Iglesia en el NT.

El previo análisis es importante debido a que uno de los pilares de la teología de la última generación dice que la última generación de cristianos (los 144.000) alcanzará un nivel de santidad no logrado por las previas generaciones de creyentes ${ }^{9}$. Sin embargo, como hemos visto, la condición "sin mancha" que presentan los 144.000 no es una característica nueva ni distintiva de este grupo. Esta particularidad es un requisito de la iglesia del NT y un requerimiento alcanzado en el AT por Abraham y Job. Por lo tanto, la frase "son sin mancha" no se refiere a que los 144.000 han alcanzado una perfección absoluta; sino más bien señala que la condición de este grupo será igual a la experimentada por Abraham y Job. Estos últimos eran sin mancha, pero no impecables (Gn 12:11-13, 18-20; 16:1-4; Job 40:1-5; 42:1-6).

Finalmente, es oportuno recordar que las características de los 144.000 son adquiridas por medio de la sangre de Jesús, ya que ellos "han lavado sus ropas y las han blanqueado en la sangre del Cordero" (Ap 7:14). Esta escena nos recuerda al Israel literal que previo a su liberación del pecado en Egipto tuvieron que emplear la sangre del cordero como medio de salvación (Ex 12:1-13 Cf. Heb 9:23-28). Por esta razón, las cualidades de los 144.000 dependen de su relación con el Cordero.

\section{Elena G. de White y los 144.000}

Esta sección analizará brevemente solo dos textos de Elena G. de White. El primero de ellos nos habla acerca del contexto histórico en donde los 144.000 se constituyen como grupo, mientras que el segundo pasaje presenta la relación de este grupo con la gran multitud.

\section{La formación de los 144.000}

El primer texto lo encontramos en los comentarios de Elena G. de White en el Comentario Bíblico Adventista, tomo 7 A. Allí la autora dice lo siguiente:

El Señor me ha mostrado claramente que la imagen de la bestia será formada antes que termine el tiempo de gracia, porque constituirá la gran prueba para el pueblo de Dios por medio de la cual se decidirá el destino de cada uno (Apocalipsis 13:11-17). Esta es la prueba que deberán enfrentar los hijos de Dios antes de ser sellados (NICHOL et al., 1999, p. 987) ${ }^{10}$.

\footnotetext{
9 Para ver una descripción completa de las ideas sobre la teología de la última generación véase (STEFANOVIĆ, 2018).

10 El énfasis es mío.
} 
Este texto nos presenta dos elementos importantes: (1) nos dice que el pueblo de Dios debe pasar por una gran prueba antes de ser sellado, probablemente la "gran tribulación" de la cual ha salido la gran multitud (Ap 7:14) ${ }^{11}$. Si este último pensamiento es correcto, entonces este texto nos dice que ambos grupos son uno, ya que son los 144.000 quienes reciben el sello antes que "la tierra, el mar y los árboles" sean dañados. (2) El pasaje de Elena G. de White nos dice que los 144.000 se constituirán como grupo cuando "termine el tiempo de gracia", momento en donde ellos serán sellados. Esta idea estaría en armonía con lo que la Biblia menciona al situar a los 144.000 en la última etapa de este mundo, previo a las siete últimas plagas y a la segunda venida de Cristo ${ }^{12}$.

\section{Los 144.000 y la gran multitud}

En este segundo texto Elena G. de White no solo destaca las conclusiones del análisis previo, sino también, parece enfatizar la conexión entre ambos grupos. Debido a lo extenso del texto, se presentarán solo los principales párrafos; el lector puede consultar la versión completa de la cita en el libro el Conflicto de los Siglos desde la página 411.

En la primera parte de la cita, Elena G. de White presenta una descripción del grupo que más tarde comenzará a presentar en detalle. Ella comenta que estos son aquellos que ha alcanzado la victoria, "sobre la bestia y su imagen, y su marca y el número de su nombre". Continúa diciendo que este grupo son los 144.000, que se encuentran en el monte Sion con las "arpas de Dios"13. Ella sigue explicando que ellos, "Cantan 'un cántico nuevo' delante del trono, un cántico que nadie podía aprender salvo los 144.000” (WHITE, 2007, p. 411) (Cf. Ap 14:3). Luego enumera algunas características de este grupo diciendo que ellos son, "los que siguen al cordero por donde quiera que va [...] [son] primicias para Dios y para el Cordero" (WHITE, 2007, p. 411) - todas ellas características relacionadas con los 144.000 (Ap 14:4). Sin embargo, en su texto Elena G. de White enumera cualidades de la gran multitud para describir a los 144.000; tal como se puede ver en el siguiente párrafo:

\footnotetext{
Estos [los 144.000] son los que han salido de la gran tribulación; han soportado el tiempo de angustia cual nunca fue desde que hubiera nación; han soportado la angustia del tiempo de angustia de Jacob; han estado sin un intercesor durante el derramamiento final de los juicios de Dios. Pero han sido librados, pues han lavado sus ropas, y las han emblanquecido en la sangre del Cordero. En su boca no fue hallada mentira pues son sin mancha delante de Dios. Por eso están delante del trono de Dios, y le sirven día y noche en su templo [...]. Han visto la tierra asolada con hambre y pestilencia, al sol que tenía poder de quemar a los hombres con un intenso calor, y ellos mismos han soportado sufrimientos, hambre y sed. Pero ya no tendrán hambre ni sed, y el sol no caerá más sobre ellos, ni calor alguno; porque el Cordero que está en medio del trono los pastoreará, y los guiará a fuentes de aguas de vida; y Dios enjugará toda lágrima de los ojos de ellos (WHITE, 2007, p. 411-412).
}

\footnotetext{
11 Véase la discusión bajo el título "Han salido de la gran tribulación".

12 Véase la discusión bajo el título "El contexto en el que se haya este grupo".

13 Véase la relación entre los 144.000 y la imagen de la bestia, la cursiva es mía.
} 
Lo interesante de este texto, es que al parecer para Elena G. de White los 144.000 y la gran multitud son un mismo grupo, puesto que, al describir al primero ella menciona cualidades que la Biblia le atribuye solo a la gran multitud, tal como se aprecia en el siguiente cuadro.

Cuadro 1

\begin{tabular}{|l|l|l|}
\hline Elena de White y los $\mathbf{1 4 4 . 0 0 0}$ & Los $\mathbf{1 4 4 . 0 0 0}$ en la Biblia & La gran multitud en la Biblia \\
\hline Están en el monte Sion & Ap 14:1 & \\
\hline Cantan un cántico nuevo & Ap 14:3 & \\
\hline Siguen al Cordero & Ap 14:4a & Ap 7:14 \\
\hline Son las primicias & Ap 14:4b & Ap 7:14b \\
\hline Vienen de la gran tribulación & & \\
\hline Lavan sus ropas en la sangre del & & Ap 7:15 \\
\hline Cordero & Ap 14:5 & Ap 7:16a \\
\hline No mienten, son sin mancha & & Ap 7:16a \\
\hline Sirven en el templo de Dios & & Ap 7:16b \\
\hline Han sufrido hambre y sed & & Ap 7:17 \\
\hline No tendrán hambre ni sed & & Ap 7:17b \\
\hline El sol no caerá más sobre ellos & & \\
\hline El Cordero los pastoreará & & \\
\hline Dios enjugará sus lágrimas & & \\
\hline
\end{tabular}

Fuente: elaboración propia

Tal como se muestra en el cuadro, Elena G. de White comienza su descripción enumerando cualidades relacionadas con los 144.000. Sin embargo, ella intercala las descripciones de este grupo con las de la gran multitud. La pregunta es ¿por qué hace esto? La respuesta parece ser que para ella ambos grupos son uno, conclusión que está en armonía con el análisis de los textos bíblicos presentado en este estudio. No obstante, es importante recordar que debido a la cantidad de interpretaciones sobre los 144.000 en la Iglesia Adventista del Séptimo Día, no podemos ser dogmáticos en nuestras conclusiones. En este sentido, es fundamental recordar las palabras de Elena G. de White: "No es su voluntad que entren en controversias por cuestiones que no los ayudarán espiritualmente, tales como: ¿Quiénes han de componer los 144.000? Fuera de duda, esto lo sabrán dentro de poco los que sean elegidos de Dios" (WHITE, 1992, p. 273).

\section{Consideraciones finales}

De acuerdo al análisis presentado en este estudio, los 144.000 deben ser interpretados como un número simbólico por las siguientes razones: 
1. Sus características: (a) ellos son el Israel espiritual, aquellos que pertenecen a "las tribus de los hijos de Israel", expresión común en el NT para referirse a la iglesia cristiana en su conjunto; (b) son un grupo redimido/comprado "de entre los de la tierra", por medio de la sangre del Cordero; (c) no se contaminan con las "mujeres/rameras" y sus falsas doctrinas, manteniéndose fieles a Jesús; (d) son "sin mancha", pues dependen de su estrecha relación con el Cordero.

2. Su relación con la gran multitud, en donde se destaca: (a) la técnica literaria "oí y vi", Juan oye el número 144.000 y luego ve una gran multitud; (b) ambos grupos están relacionados por medio de la frase "han salido de la gran tribulación", la prueba final que tendrá que enfrentar la iglesia al oponerse a los enemigos de Dios; (c) la conexión entre los 144.000, la gran multitud y las características del Cordero. Apocalipsis 7 presenta estrechas conexiones con el capítulo 5, lugar en donde el León está relacionado con la primera de las tribus de Israel; mientras que el Cordero presenta conexiones con la gran multitud.

3. Los textos de Elena G. de White, en donde al parecer ella apoya las conclusiones anteriores comentando que: (a) los 144.000 serán un grupo que se constituirá en la etapa final de este mundo, momento en donde deberán pasar por una gran prueba relacionada con los enemigos de Dios. Esta gran prueba probablemente se refiere a la gran tribulación de la cual a salido la gran multitud; (b) Elena G. de White atribuye cualidades de la gran multitud a los 144.000, lo que podría deberse a que ella considera que ambos son el mismo grupo.

Los 144.000 y la gran multitud son el mismo grupo presentado en diferentes escenarios, mientras que el primero señala a la "iglesia militante", el segundo representa a la "iglesia triunfante". Este es un grupo especial de redimidos, los cuales recibirán el sello de Dios para enfrentar la "gran tribulación". Es en medio de esta gran aflicción espiritual, en donde los 144.000 serán liberados por el cordero Jesús, quien los ha redimido/comprado con su sangre (Ap 14:14). Este último evento nos recuerda la salvación del pueblo de Dios en Egipto, siendo liberados por medio de la sangre del cordero (Ex 12:1-13). Apocalipsis narra que ese acontecimiento pasado se volverá a repetir, la iglesia será liberada de la Babilonia espiritual por medio de la sangre del cordero Jesús. Los hijos de Dios no tienen nada que temer, ya que en el momento de mayor aflicción Miguel se levantará para rescatar a su pueblo (Dn 12:1 Cf. Ap 16:12; 19:11-21).

\section{Bibliografía}

BAUCKHAM, R. The climax of prophecy: studies on the book of revelation. Edinburgh: Clark, 1998.

BEALE, G. K. Revelation: a Shorter Commentary. Grand Rapids, MI: William B. Eerdmans Publishing, 2015.

MENDOZA, O. Los 144000 y la gran multitud en el contexto de Apocalipsis 7 y 14. Theologika, v. 26, n. 1, p. 49-82, 2011.

MOUNCE, R. H. The Book of Revelation. Grand Rapids, MI: W.B. Eerdmans, 1998. 
MOUNCE, W. D. (Ed.). Mounce's complete expository dictionary of Old \& New Testament words. Grand Rapids, MI: Zondervan, 2006.

MUELLER, E. Fornication. Biblical Research Institute. 2001. Disponível em: <https://bit. ly/3r03yDn>. Acesso em: 16 fev. 2020

NICHOL, F. D.; RASI, H. M.; PEVERINI, T. N.; HORN, S. H. Comentario bíblico Adventista del Séptimo Día 7: Filipenses a Apocalipsis. Mountain View, California: Pacific Press Publishing Association, 1995.

NICHOL, F. D.; RASI, H. M.; PEVERINI, T. N.; HORN, S. H. Comentario bíblico Adventista del Séptimo Día 7A: Comentarios de E. G. de White. Mountain View, California: Pacific Press Publishing Association, 1999.

STEFANOVIĆ, R. La Revelación de Jesucristo: comentario del libro del apocalipsis. Berrien Springs, MI: Andrews University Press, 2013.

STEFANOVIĆ, R. What is the State of the Last Generation? In: MOSKALA, J.; PECKHAM, J. (Eds.). God's character and the last generation. Nampa, ID: Pacific Press Publishing Association, 2018. p. 219-235.

WHITE, E. G. El conflicto de los siglos. Buenos Aires, Argentina: ACES, 2007. 